NASA Technical Memorandum 102746

NASA-TM-102746 19910002802

\title{
THERMAL-STRUCTURAL FINITE ELEMENT ANALYSIS USING LINEAR FLUX FORMULATION
}

\section{AJAY K. PANDEY \\ PRAMOTE DECHAUMPHAI \\ ALLAN R. WIETING}

OCTOBER 1990

\section{N/SN \\ National Aeronautics and \\ Space Administration \\ Langley Research Center \\ Hampton, Virginia 23665}

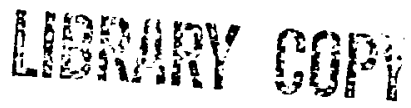

:UV 191990

LANGLEY RESEARCH CENTER

W HAMPTON, VIRGINIA 


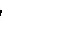




\title{
THERMAL-STRUCTURAL FINITE ELEMENT ANALYSIS USING LINEAR FLUX FORMULATION
}

\author{
Ajay K. Pandey* \\ Planning Research Corporation \\ Hampton, Virginia \\ Pramote Dechaumphai** and Allan R. Wieting*** \\ NASA Langley Research Center \\ Hampton, Virginia
}

\section{Abstract}

A linear flux approach is developed for a finite element thermal-structural analysis of steady-state thermal and structural problems. The element fluxes are assumed to vary linearly in the same form as the element unknown variables, and the finite element matrices are evaluated in closed form. Since numerlcal integration is avoided, significant computational time saving is achieved. Solution accuracy and computational speed improvements are demonstrated by solving several two- and three-dimensional thermalstructural examples.

\section{Nomenclature}

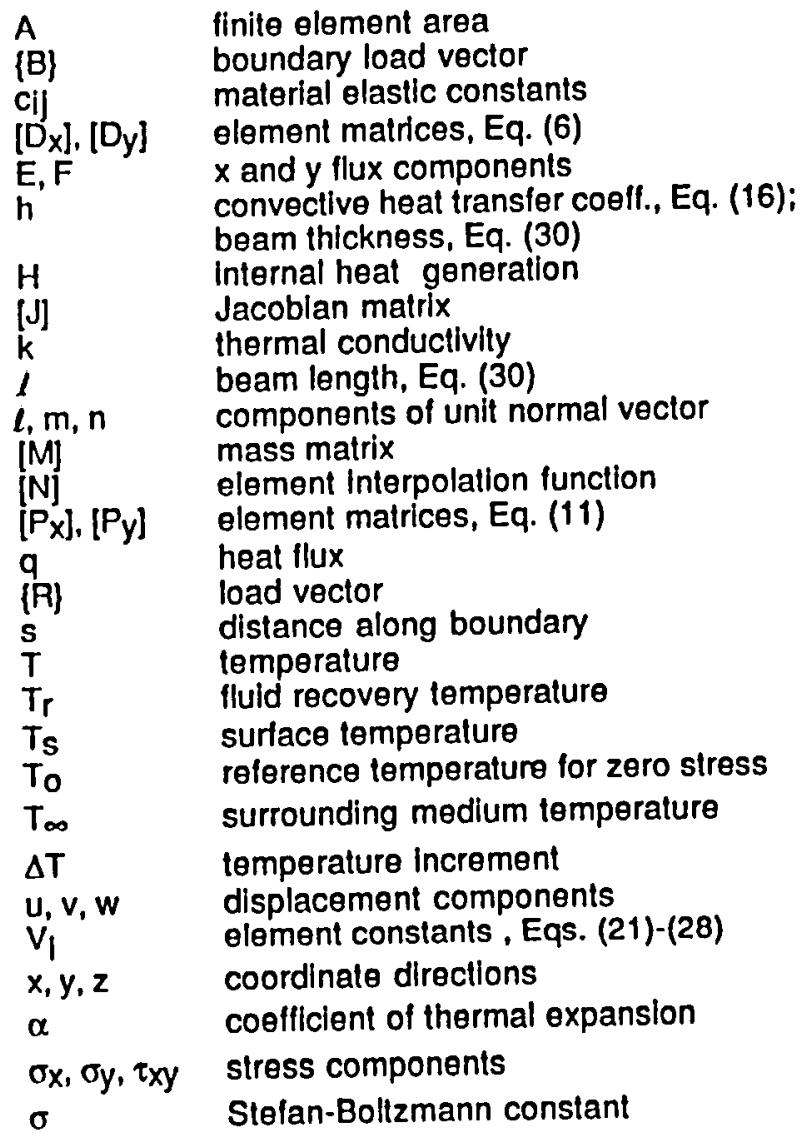

\footnotetext{
"Project Struclures Engineer, Aerospace Technologies Dlvision, Member AIAA.

"Aerospace Technologisl, Aerolhermal Loads Branch, Member AIAA.

A.Head, Aerothermal Loads Branch, Member AlAA.
}

\author{
$\varepsilon \quad$ strain components, Eq. (15); \\ emissivity, Eq. (16)
Subscripts
$T$ thermal
s structural \\ Superscripts \\ T transpose

\section{Introduction}

For hypersonic vehicles to become a practical reality, efficient techniques are needed to analyze light weight airframe and engine structures for repeated and prolonged exposure to their severe flight environment. To understand the structural response under these severe aerodynamic loads, research in the Aerothermal Loads Branch, NASA Langley Research Center has focused on developing effective computational approaches for predicting the aerodynamic flow and the thermal and structural response of the structure, including their interactions ${ }^{1}$. The approaches consist of using: (1) a general automated unstructured gridding to discretize the aerodynamic flow field and the structure, (2) finite element methods to solve for the environment, loads, and response for all three disciplines (flow, thermal and structural response), and (3) adaptive mesh refinement techniques with error indicators to minimize the number of grid points and increase the solution accuracy.

A Taylor-Galerkin finite element algorithm, has been used recently to predict the aerodynamic flow field as well as the thermal-structural response for high speed flow over leading edges 2 . The approach utilizes: (1) a Taylor series expansion in time to establish recurrence relations for time marching, and (2) the method of weighted residuals with Galerkin's criterion for spatial discretization. The governing equations are cast in conservation form. The standard primitive variables are replaced with their flux counterparts, which are assumed to vary linearly over the elements. This formulation allows the finite element matrices to be evaluated in closed form, thereby avoiding the more expensive numerical integration. Since the TaylorGalerkin algorithm is a time marching (transient) algorithm, the full benefits of the linear flux formulation for steady-state problems (steady-state heat transfer and static structural problems) has not been exploited. Furthermore, most structural problems may be treated as quasi static even when the aerodynamic loads and the thermal response are transient.

The purpose of this paper is to extend the TaylorGalerkin algorithm to steady-state thermal-structural 
analysis. The linear flux formulation and solution procedure are introduced. The finite element matrices (in integral forms) which are different from those appearing in the conventional finite element formulation, are presented. A method to derive these finite element matrices in closed form is developed and presented for both two- and three-dimensional elements.

The capability of the linear flux formulation is demonstrated using four examples: (1) a thermal analysis of a circular plate with internal heat generation, (2) a structural analysis of a beam-bending due to thermal load, (3) a thermal-structural analysis of a twodimensional aerodynamically heated leading edge model, and (4) a structural analysis of a thre日dimensional leading edge model. Results are compared with available analytical solutions and the conventional finite element solutions.

\section{Thermal-Structural Formulation}

The derivation of finite element equations using a linear flux formulation is presented for steady-state thermal and structural analyses. For simplicity, the derivation presented herein is for two-dimensional problems. Extension to three-dimensional problems is straightforward. The governing equations are written in conservation form so that the linear flux formulation can be used directly. This formulation yields finite element matrices which can be evaluated in closed form. A method to evaluate these closed form matrices is described for both two- and three-dimensional elements.

\section{Governing Equations}

Heat Transfer. The steady-state thermal response of a structure is governed by the energy equation in conservation form,

$$
\frac{\partial}{\partial x}\left(E_{T}\right)+\frac{\partial}{\partial y}\left(F_{T}\right)=H_{T}
$$

where the subscript $T$ denotes the thermal analysis, $\mathrm{E}_{T}$ and $F_{T}$ are the heat flux components, and $H_{T}$ is the heat source per unit volume. The heat fluxes $E_{T}$ and $F_{T}$ are related to temperature gradients by Fourier's law.

Structural Response. The static structural response is governed by the equilibrium equations in conservation form,

$$
\frac{\partial}{\partial x}\left\{E_{s}\right\}+\frac{\partial}{\partial y}\left\{F_{s}\right\}=0
$$

where the subscript $s$ denotes the structural analysis. The vectors $\left\{E_{s}\right\}$ and $\left\{F_{s}\right\}$, which contain the stress components, are given by

$$
\begin{aligned}
& \left\{E_{s}\right\}^{\top}=\left[\begin{array}{ll}
\sigma_{x} & \tau_{x y}
\end{array}\right] \\
& \left\{F_{s}\right\}^{\top}=\left[\begin{array}{ll}
\tau_{x y} & \sigma_{y}
\end{array}\right]
\end{aligned}
$$

The stress components $\sigma_{x}, \sigma_{y}$, and $\tau_{x y}$ are related to the displacement gradients and the temperature by the generalized Hooke's law.

\section{Solution Procedure}

For simplicity in presenting the linear flux algorithm, both the steady-state energy equation and structural equilibrium equations are written in the form of a scalar equation as,

$$
\frac{\partial E}{\partial x}+\frac{\partial F}{\partial y}=H
$$

Even though the derivation presented below is for the thermal analysis, the procedure can be applied directly to the structural analysis.

LInear Flux Assumptions. The key feature of the linear flux formulation in the thermal analysis is to assume the distribution of the element heat fluxes $E$ and $F$ in the same form as the element temperalure distribution $T$, that is

$$
\begin{array}{lll}
T(x, y) & = & {[N(x, y)]\{T\}} \\
E(x, y) & = & {[N(x, y)]\{E\}} \\
F(x, y) & = & {[N(x, y)]\{F\}}
\end{array}
$$

where $[N(x, y)]$ are the element interpolation functions, and $\{T\},\{E\}$ and $\{F\}$ are the vectors of the element nodal quantities. The assumption of a linear distribution of element fluxes $E$ and $F$ which are interpolated in the same form as other dependent variables (e.g. T, as shown in Eq. (5)), is widely used in the computational fluid dynamics ${ }^{2}$.

Finite Element Equations. The finite element equations are derived using the method of weighted residuals ${ }^{3}$. The governing differential equation, Eq. (4), is multiplied by the weighting functions, $[N(x, y)]$, and integrated over the element area $A$. Integration by parts is performed to produce element integral terms and the boundary surface integral terms for application of different types of thermal boundary conditions. Detalls of the derivation follows the conventional finite element approach described in Ref. 3. The finite element equations obtained are in the form,

$$
\left[D_{x}\right]\{E\}+\left[D_{y}\right]\{F\}+\{A\}+\{B\}=0
$$

In this equation, the matrices $\left[D_{x}\right]$ and $\left[D_{y}\right]$ are

$$
\begin{aligned}
& {\left[D_{x}\right]=\int_{A}\left\{\frac{\partial N}{\partial x}\right\}[N] d A} \\
& {\left[D_{y}\right]=\int_{A}\left\{\frac{\partial N}{\partial y}\right\}[N] d A}
\end{aligned}
$$

The element nodal vector. $\{R\}$, associated with the heat source, $H$, is defined as

$$
\{R\}=\int_{A}\{N\} H d A
$$

The vector $\{B\}$ representing the boundary nodal vector is defined as 


$$
\begin{aligned}
\{B\} & =\int_{8}\{N\}[N] d s(I\{E\}+m\{F\}) \\
& =\int_{s}\{N\}[N] d s\{q\}
\end{aligned}
$$

where $l$ and $m$ are the components of a unit vector normal to the element boundary. The finite element matrices shown in Eqs. (7)-(9) are evaluated in closed form as will be demonstrated in the subsequent seclion.

Since the fluxes, $E$ and $F$, are related to the temperature gradients glven by Fourier's law,

$$
\begin{aligned}
& E=-k \frac{\partial T}{\partial x} \\
& F=-k \frac{\partial T}{\partial y}
\end{aligned}
$$

where $k$ is the material thermal conductivity, the element nodal flux vectors, $\{E\}$ and $\{F\}$, can be expressed in terms of element nodal temperature, $\{T\}$, as

$$
\begin{aligned}
& \{E\}=-k\left[P_{x}\right]\{T\} \\
& \{F\}=-k\left[P_{y}\right]\{T\}
\end{aligned}
$$

where the matrices, $\left[P_{x}\right]$ and $\left[P_{y}\right]$, are related to the element shape and are given in the Appendix. The element nodal flux vectors, Eqs. (11a) and (11b), are substituted into Eq. (6) to obtain the final finite element equations in terms of the unknown element nodal temperature, $\{T\}$, in the form,

$$
[K]\{T\}=\{R\}+\{B\}
$$

where the stiffness (or conduction) matrix, $[\mathrm{K}]$, is given by

$$
[K]=k\left[D_{x}\right]\left[P_{x}\right]+k\left[D_{y}\right]\left[P_{y}\right]
$$

Equation (12) is in a form similar to that obtained from the conventional finite element approach except that the latter stillness matrix is defined by

$$
\begin{aligned}
{[\bar{K}]=} & \int_{A} k\left\{\frac{\partial N}{\partial x}\right\}\left[\frac{\partial N}{\partial x}\right] d A+ \\
& \int_{A} k\left\{\frac{\partial N}{\partial y}\right\}\left[\frac{\partial N}{\partial y}\right] d A
\end{aligned}
$$

Evaluation of the conventional stiffness matrix, $[\bar{K}]$, for some element types, such as the two-dimensional quadrilateral and three-dimensional hexahedral elements, requires the use of numerical integration.

For nonlinear problems (e.g. due to temperature dependent thermal conductivity). Eq. (12) is solved by the Newton-Raphson iteration technique 3,4. This procedure is identical to that used in the conventional finite element approach.

The approach presented for the thermal analysis is applied directly to derive the finite element equations for the structural analysis. The equations are identlcal to Eqs. (5)-(13), where the temperature vector, $\{T\}$, is replaced by the displacement vector conlaining the components $u$ and $v$ in $x$ - and $y$-directions, and the vectors $\{E\}$ and $\{F\}$ represent the element nodal stress components. For elastic orthotropic materials, typical nodal stress components in two dimensions are obtained using the constitutlve relations,

$$
\sigma_{1}=c_{i j} \varepsilon_{1}+\beta_{1}\left(T-T_{0}\right) \quad i, j=1,2,3
$$

where $\varepsilon_{j}$ are the nodal strain components, and $T_{0}$ is the reference temperature for zero thermal stress. The material elastic constants, cij, and the thermal expansion parameter, $\beta i$, may be temperature dependent.

Boundary Conditions. The boundary conditions for thermal analysis are applied via the boundary nodal vector $\{B\}$ shown in Eq. (9). The vector $\{\mathrm{q}\}$ appearing in this equation may be replaced by different types of boundary conditions,

$$
q= \begin{cases}0 & \text { (insulated) } \\ q_{s} & \text { (specified heating) } \\ h\left(T_{s}-T_{s}\right) & \text { (surface convection) } \\ \varepsilon \sigma\left(T_{s}^{4}-T_{\infty}^{4}\right) & \text { (surface radiation) }\end{cases}
$$

The boundary conditions for the structural analysis, such as the applied surface pressure, can be added into the structural equations via the surface boundary vector. The procedure is identical to that for the thermal analysis previously described and is therefore omitted.

\section{Derivation of Closed Form Flnite Element Matrlces and Element Nodal Gradlents}

All tinite element integrals, such as [Dx], [Dy], and $\{R\}$, which are given by Eqs. (7) and (8), can be expressed in closed forms. This is true for simple element types (rods, triangles and tetrahedrons) as well as for more popular elements (quadrilaterals and hexahedrons). In addition, closed form expressions for other finite element integrals (such as the consistent mass matrix) and the gradients of element variables (such as $\partial T / \partial x$ ) which are normally required in other finite element formulations can be obtained by the procedure described below.

\section{Quadrllateral Element}

Typical finite element integrals for a general quadrilateral element, as shown in Fig. 1, are given below using natural coordinates

$$
\begin{aligned}
& {[M]=\int_{-1}^{1} \int_{-1}^{1}[N]^{\top}[N]|J| d \xi d \eta} \\
& {\left[D_{x}\right]=\int_{-1}^{1} \cdot \int_{-1}^{1}\left\{\frac{\partial N}{\partial x}\right\}[N]|J| d \xi d \eta}
\end{aligned}
$$

where $[M]$ is the consistent mass matrix and $\left[D_{X}\right]$ is the element matrix previously defined in Eq. (7a). The determinant of the Jacobian $|J|$ represents the transformation from the element global $x-y$ coordinates 
to the natural coordinates $\xi-\eta$ (see Fig. 1). The transformation permits the element integration to be evaluated over a square. The determinant of the Jacobian for the two-dimensional quadrilateral element is

$$
\begin{aligned}
|J| & =\left|\begin{array}{lr}
J_{11} & J_{12} \\
J_{21} & J_{22}
\end{array}\right| \\
& =\left|\begin{array}{cc}
\frac{\partial}{\partial \xi} \sum_{i=1}^{4}\left(N_{1} x_{1}\right) & \frac{\partial}{\partial \xi} \sum_{i=1}^{4}\left(N_{1} y_{1}\right) \\
\frac{\partial}{\partial \eta} \sum_{i=1}^{4}\left(N_{1} x_{1}\right) & \frac{\partial}{\partial \eta} \sum_{i=1}^{4}\left(N_{1} y_{1}\right)
\end{array}\right| \\
& =\frac{\partial}{\partial \xi} \sum_{i=1}^{4}\left(N_{1} x_{1}\right) \cdot \frac{\partial}{\partial \eta} \sum_{i=1}^{4}\left(N_{1} y_{1}\right)
\end{aligned}
$$

where

$$
N_{1}=N_{1}(\xi, \eta)=\frac{1}{4}\left(1+\xi \xi_{i}\right)\left(1+\eta \eta_{1}\right) \quad i=1, \ldots, 4
$$

The algebraic expression for the determinant of the Jacobian shown in Eq. (19) is in the form of the partial derivatives of the element interpolation functions $N_{i}(\xi, \eta)$ and the element nodal locations $\left(x_{i}, y_{i} ; l=1, \ldots, 4\right)$. The expression for the determinant of the Jacobian is quite lengthy (contains a total of 64 terms if fully expanded), and thus results in a tedious task for deriving the closed form element matrices (Eq. (17) and (18)). Such a task becomes almost impossible for the threedimensional 8-node hexahedral element in which the determinant of the Jacobian, if fully expanded, contains approximately 200,000 terms. To overcome this difficulty, the determinant of the Jacobian is rewritten in an alternate simpler form,

$$
|J|=\Sigma \bar{N}_{1}(\xi, \eta) V_{1}
$$

where $\bar{N}_{i}(\xi, \eta)$ are the functions of the natural coordinates $\xi$ and $\eta$, and are selected so that Eq. (21) represents the complete order of polynomials of $\xi$ and $\eta$ as appearing in the original Eq. (19). The unknown constants, $V_{i}$, are functions of the nodal coordinates and are to be determined. For a two-dimensional quadrilateral element, the functions, $\bar{N}_{i}(\xi, \eta)$, can be represented by the isoparametric quadrilateral element interpolation functions, $\mathrm{Ni}_{i}(\xi, \eta)$, as given by $\mathrm{Eq}$. (20). Equation (21) then becomes

$$
\begin{aligned}
|J| & =\sum_{i=1}^{4} N_{1}(\xi, \eta) V_{1} \\
& =N_{1} V_{1}+N_{2} V_{2}+N_{3} V_{3}+N_{4} V_{4}
\end{aligned}
$$

The unknowns, $v_{i}, i=1, \ldots, 4$, can then be determined easily using the properties of $N_{i}(\xi, \eta)$, that is $N_{i}(\xi, \eta)$ equal to unity at node $I$ and zero at the other nodes. As an example, $V_{1}$ is obtalned by equating Eqs. (22) and (19) with the property at node $1(\xi=\eta=-1$ in Fig. 1) to give

$v_{1}=\left[\left(x_{2}-x_{1}\right) y_{4}+\left(x_{1}-x_{4}\right) y_{2}+\left(x_{4}-x_{2}\right) y_{1}\right] / 4$

The use of the determinant of the Jacobian in the form of Eq. (21) instead of the original Eq. (19) permits the finite element matrices, (Eq. (17) and (18)), to be evaluated in closed forms. The use of the symbolic manipulation program MACSYMA5 greatly simplified this evaluation.

The determinant of the Jacobian in the form of Eq. (21) is also used to derive closed form expressions for the element gradients. For example, the element temperature gradient, $\partial T / \partial x$, is given by

$$
\frac{\partial T}{\partial x}=\frac{1}{|J|}\left(J_{22}\left\lfloor\frac{\partial N}{\partial \xi}\right\rfloor-J_{12}\left\lfloor\frac{\partial N}{\partial \eta}\right\rfloor\right)\{T\}
$$

where $\mathrm{J}_{12}$ and $\mathrm{J}_{22}$ are defined in Eq. (19). The temperature gradient at node 1 can be determined by setting $\xi=\eta=-1$ to yield

$$
\left.\frac{\partial T}{\partial x}\right|_{\text {node } 1}=\frac{\left(T_{2}-T_{1}\right) y_{4}+\left(T_{1}-T_{4}\right) y_{2}+\left(T_{4}-T_{2}\right) y_{1}}{\left(x_{2}-x_{1}\right) y_{4}+\left(x_{1}-x_{4}\right) y_{2}+\left(x_{4}-x_{2}\right) y_{1}}
$$

where subscripts denote the element node numbers shown in Fig. 1.

The approach presented here is used to derive closed form expressions for the other finite element matrices (eg. [Dy], and $\{R\})$ and nodal variable gradients. These closed form expressions are used in the formulation for both thermal and structural analyses.

\section{Hexahedral Element}

The three-dimensional finite element matrices are in the same form as shown in Eqs. (17) and (18), except that integration is performed over the element volume. For an 8-node hexahedral element as shown in Fig. 2, the element interpolation functions are,

$$
N_{1}=N_{1}(\xi, \eta, \zeta)=\frac{1}{8}\left(1+\xi \xi_{1}\right)\left(1+\eta \eta_{1}\right)\left(1+\zeta \zeta_{i}\right) \quad l=1, \ldots, 8
$$

and the determinant of the Jacobian is given by,

$$
|J|=\left|\begin{array}{lll}
\frac{\partial}{\partial \xi}\left(\Sigma N_{1} x_{1}\right) & \frac{\partial}{\partial \xi}\left(\Sigma N_{1} y_{1}\right) & \frac{\partial}{\partial \xi}\left(\Sigma N_{1} z_{1}\right) \\
\frac{\partial}{\partial \eta}\left(\Sigma N_{1} x_{1}\right) & \frac{\partial}{\partial \eta}\left(\Sigma N_{1} y_{1}\right) & \frac{\partial}{\partial \eta}\left(\Sigma N_{1} z_{1}\right) \\
\frac{\partial}{\partial \zeta}\left(\Sigma N_{1} x_{1}\right) & \frac{\partial}{\partial \zeta}\left(\Sigma N_{1} y_{1}\right) & \frac{\partial}{\partial \zeta}\left(\Sigma N_{1} z_{1}\right)
\end{array}\right|
$$

By following the procedure described in the previous section, the above determinant of the Jacobian is rewritten in an alternate simpler form as in Eq. (21). In three dimensions, the hexahedral element interpolation functions, $\mathrm{Ni}_{i}(\zeta, \eta, \zeta)$, given In Eq. (26), can not be used to represent the function, $\bar{N}_{i}(\xi, \eta, \zeta)$, in the same fashion as in the two-dimensional Jacobian formulation. This is because the hexahedral element interpolation functions, 
$N_{i}(\xi, \eta, \zeta)$, do not provide a complete polynomial as required by the determinant of the Jacoblan shown In Eq. (27). Therefore the functions, $\bar{N}_{i}(\xi, \eta, \zeta)$, are represented by 27-node Langrange cubic element interpolation functions to obtain a simpler form for the Jacobian determinant, given as Eq. (28)

$$
|J|=\sum_{i=1}^{27} N_{i}(\xi, \eta, \zeta) V_{1} \quad i=1, \ldots, 27
$$

The unknowns $V_{\mathbf{j}}$ are determined by equating the Eqs. (27) and (28) at the nodal locations of the Lagrange cubic element. The program MACSYMA5 was used to derive these unknowns, $V_{l}$, as well as the associated element matrices and the element nodal gradients in closed form. The algebraic expressions for the matrices and the gradients were very lengthy therefore they were translated into FORTRAN statements using MACSYMA and were used directly in the analysis code. The use of these closed form expressions reduces the computational time compared to the traditional numerical integration, as will be demonstrated in the next section.

\section{Application}

Four examples are presented to demonstrate the accuracy and computational efficiency of the linear flux formulation. These consist of: (1) a thermal analysis of a circular plate with internal heat generation, (2) a structural analysis of a beam bending due to thermal load, (3) a two-dimensional thermal-structural analysis of an aerodynamically heated leading edge model, and (4) a three-dimensional structural analysis of an aerodynamically heated leading edge model. Results obtained by the linear flux formulation are compared with available analytical solutions and the conventional finite element solutions.

\section{Clrcular Plate With Internal Heat Generation}

A 20 in. diameter stainless steel circular plate with internal heat generation and specified zero temperature along the circumferential boundary shown in Fig. 3 is used as the first example. Analytical solution for the variation of temperature in the plate is available and is given by

$$
T(r)=\frac{Q}{4 k}\left(100-r^{2}\right)
$$

where $Q$ is uniformly distributed internal heat generation , rate per unit volume, $k$ the plate thermal conductivity and $r$ the plate radial distance. Due to symmetry, only a quarter of the plate is modeled with 10 quadrilateral elements as shown in Fig. 3. The distorted quadrilateral element shape was selected to evaluate the formulation performance under an arbitrary unstructured mesh condition.

The plate temperature distribution obtained from the linear flux formulation is compared with the analytical solution (Eq. (29)) and the conventional finite element solution in Fig. 4. Because of the crude mesh used in the model, both finite element solutions underpredict the temperature distribution. The temperatures at nodes $A, B$, and $C$ (see Fig. 3), obtained by the analytical method, Innear flux approach, and the conventional approach are shown in Table 1 . These results indicate that the linear flux formulation provides slightly higher solution accuracy compared to the conventional finite element formulation. This is due to the fact that four Gauss point numerical Integration, which is commonly used to evaluate the conventional finite element stiffness matrix, (see Eq. (14)), can not provide exact integration for arbitrary quadrilateral element shapes.

\section{Table 1 Comparative nodal temperature ( $\mathrm{OF}$ )} and errors

\begin{tabular}{lccccc}
\hline Location & $\begin{array}{c}\text { Analytical } \\
\text { Eq. (29) }\end{array}$ & $\begin{array}{c}\text { Linear flux } \\
\text { Temp. }\end{array}$ & $\begin{array}{c}\text { Conventional } \\
\%\end{array}$ & & $\begin{array}{c}\text { Tomp. } \\
\text { Terror } \\
\%\end{array}$ \\
\hline A & 100.0 & 96.9 & 3.1 & 96.5 & 3.5 \\
B & 92.0 & 88.3 & 4.0 & 87.9 & 4.4 \\
C & 68.0 & 67.8 & 0.3 & 67.3 & 1.0 \\
\hline
\end{tabular}

The computational time for linear flux approach and the conventional approach are given in Table 2. The comparison of the computalional time Indicates a $39 \%$ savings for the linear flux approach. The time savings is due to the use of the closed form algebraic expresslons rather than the numerical Integration to evaluate the finite element stiffness matrix.

Table 2 Comparative CPU time (CRAY-2 seconds) for evalualing element stiffness matrix

\begin{tabular}{llc}
\hline \hline Linear flux & Conventional & $\%$ Saving \\
\hline $0.1036 \times 10^{-3}$ & $0.1690 \times 10^{-3}$ & 39 \\
\hline
\end{tabular}

Beam Bending Due to Thermal Load

As a second example, a $4 \mathrm{ln}$. long, $0.1 \mathrm{ln}$. thick stainless steel beam pinned on the bottom edges is considered and is shown in Fig. 5. The beam is assumed to be flat, and stress free at room temperalure. The beam temperature is raised uniformly by $65^{\circ} \mathrm{F}$. The edge constraints cause the beam to bend Into a convex shape. At this relatively low temperature and small deformation, beam structural response may be approximated by the beam-column theory 6 , in which shear effects are neglected. For cyllndrical bending, flexural rigidity of the beam, D, is equal to Eh3/12, where $E$ is the modulus of elastlcity and $h$ is the thickness of the beam (see Fig. 5). The deflection, $v(x)$, is given by 7

$$
v(x)=\frac{h}{2}\left(\frac{\cos \lambda x}{\cos \frac{\lambda l}{2}}-1\right)
$$

where $l$ is length of the beam and $\lambda$ is $\sqrt{(P / D)}$. The axial constralnt force, $P$, is computed from 


$$
\begin{aligned}
& \frac{P I}{h E}+\frac{h^{2}}{2} \lambda \tan \left(\frac{\lambda l}{2}\right)+ \\
& \frac{h^{2}}{8} \frac{P}{D} \frac{\left(\frac{l}{2}-\frac{\sin \lambda l}{2 \lambda}\right)}{\cos ^{2} \frac{\lambda l}{2}}-\alpha \Delta T l=0
\end{aligned}
$$

where $\Delta T$ is the beam temperature increment.

Using symmetry, one-half of the beam is modeled with 160 quadrilateral finite elements. The finite elements are unlformly distributed with 40 elements along the beam length and 4 elements through the beam thickness. Both the linear flux and the conventional finite element approaches yield identical beam deflections. The predicted deflection distribution is compared with the solution from the beam theory, (Eq. (30)), in Fig. 6. The figure shows a very good agreement of the beam dellection distributions with the maximum difference of about $2 \%$ at the beam center $(x=0)$.

The predicted beam deflection obtained from the linear flux formulation demonstrates the capability of the approach for providing the same solution accuracy as the conventional finite element approach for the structural analysis. Of course, the computational time saving of $39 \%$ is still achieved in the evaluation of the finite element stilfness matrix.

\section{Two-Dimenslonal Leading Edge Model}

To further demonstrate the capability of the linear flux formulation for both thermal and structural analyses, the approach is applied to predict the thermal-structural response of an aerodynamically heated leading edge subjected to a high speed flow. The leading edge consists of a 0.25 in. nose diameter, 3 in. long model made of $0.1 \mathrm{in}$. thick Inconel 617 alloy as shown in Fig. 7 . The thermal boundary conditions along the outer surface consist of applied aerodynamic heating and emitted surface radiation. The leading edge is insulated along the inner surface. A schematic of the finite element model, which consisted of 508 quadrilateral elements is given in the figure. The mesh is graded with five elements through the thickness and 127 elements along the circumference. Approximately $70 \%$ of the elements lie in the 0.25 in. nose of the leading edge.

The aerodynamic heating along the leading edge outer surface and the aerodynamic flow field represented by the Mach number contours are shown in Fig. 8. These flow solutions were obtained from Rel. 8 by solving the Navier-Stokes equations. The Mach number contours indicate an unsymmetric bow shock shape from the free stream Mach 5.25 llow, which is inclined $12.5^{\circ}$ relative to the bottom of the leading edge. The aerodynamic heating rate distribution is relatively low along both lower and upper surfaces of the leading edge compared to the stagnation point healing rate. The aerodynamic heating rate on the lower surface is slightly higher than the upper surface. The aerodynamic heating increases significantly at the nose because the flow stagnates in that region.

The leading edge aerodynamic heating, shown in the Fig. 8, was predicted assuming a uniform surface temperature of $530^{\circ} \mathrm{R}$. During the transient response, the aerodynamic heating rate decreases as the leading edge temperature increases. Thus, to obtain a realistic leading edge temperature response, the specified aerodynamic heating (qs in Eq. (16)) is converted into the surface convection boundary condition $\left(h\left(T_{S}-T_{r}\right)\right.$ in Eq. (16)). It should also be noted that the change in the surface convection coefficient, $h$, with the surface temperature, $T_{S}$, is small compared to the change in the heating rate, qs.

The predicted steady-state leading edge temperature contours and the outer surface temperature distribution are compared with the conventional finite element solutions in Fig. 9. The temperature distributions obtained from both approaches are almost identical with the maximum difference of $0.2 \%$ at the nose of the leading edge where the peak temperature occurs.

The aerodynamic pressure on the leading edge and the flow pressure contours 8 are shown in Fig 10. The peak pressure occurs at the flow stagnation point on the nose and the pressure is nearly uniform on the top and bottom surfaces. This aerodynamic pressure (Fig. 10) and the leading edge temperature (Fig. 9) are used as the aerothermal loads for prediction of the leading edge structural response. The leading edge material properties, such as the modulus of elasticity and the coefficient of thermal expansion, are temperature dependent ${ }^{9}$. The finite element discretization previously used in the thermal analysis is also used for the structural analysis to eliminate the data manipulation normally required by the different analysis disciplines. The predicted tangentlal stress contours superimposed on the deformed leading edge are shown in Fig. 11. The increased leading edge temperature causes the leading edge to expand. The temperature difference between the lower and upper sections (higher temperature on the lower section) causes the leading edge to bend and rotate upward. The figure shows that the tangential stress, which is primarily caused by the temperature difference between the two sections, is relatively low. These results are in excellent agreement with the conventional finite element results indicating the validity of the linear flux formulation for the structural analysis. Again, a $39 \%$ computational time saving is achieved using the linear flux approach.

The predicted structural response obtained from the two-dimensional leading edge model is based on a plane strain assumption. The use of this assumption results in high compressive axial stresses ( $\approx 150 \mathrm{ksi})$ in the direction normal to leading edge cross-section. A three-dimensional analysis with the appropriate boundary conditions would provide a more realistic leading edge axial stress prediction. Such a structural analysis is presented in the next application.

\section{Three-Dimenslonal Leading Edge Model}

As mentioned in the theoretical formulation section, the extension of the linear flux approach to three dimensions is straightforward. The approach has been extended for both the thermal and structural analyses using the 8-node hexahedral element. The use of the hexahedral element is preferred over other threedimensional element types (such as the tetrahedral element) to reduce the computer memory required for the analysis (a hexahedral element consist of five tetrahedral elements). The purpose of presenting this application is: (1) to compare the linear flux solution with the conventional finite element solution for a threedimensional problem, (2) to demonstrate the 
computational time saving, and (3) to predict a more realistic leading edge axial stress.

The linear flux approach is applied to predict the leading edge structural response using the threedimensional model shown in Fig. 12. The mesh on the leading edge cross-section ( $x-y$ plane) is identical to the two-dimensional model described in the previous example. The mesh is extended with a total of 10 layers in the z-direction. The aerothermal loads consist of the temperature distribution (Fig. 9) and the aerodynamic pressure (Fig. 10) which are taken to be uniform in the zdirection.

The predicted axial stress contours superimposed on the deformed leading edge along the midsection (section $z=0.75$ in.) are shown in Fig. 13. This figure shows a more realistic axial stress distribution which resembles the temperature distribution. The peak compressive stress of approximately $20 \mathrm{ksi}$ (compared to $150 \mathrm{ksi}$ for $2-\mathrm{D}$ model) occurs at the nose of the leading edge where the temperature is maximum. This significant reduction occurs because there is no axial constraint as the two-dimensional case. The conventional finite element analysis solution is nearly identical with a maximum difference in the deflection at the leading edge nose of less than $0.5 \%$. The linear flux approach shows a computational time saving of $78 \%$ over the conventional finite element approach in the evaluation of the hexahedral element stiffness matrix. Such a significant computational time saving is due to the use of closed form algebraic expressions instead of the 8-Gauss point numerical integration. Of course, fewer Gauss point integration could be used at the expense of accuracy.

\section{Concluding Remarks}

A linear flux approach for tinite element thermalstructural analysis was presented. The approach employs the assumption that the fluxes vary linearly as the dependent variables over the element. Such an assumption is widely used in computational fluid dynamics. The finite element equations for steady-state thermal and structural analyses are derived. The finite element equations consist of the finite element matrices in integral form which are different from those appearing in the conventional finite element formulation. A method was developed to derive these finite element matrices in closed form and the detalls of the derivation was described. The use of the closed form algebralc expressions for evaluating the finite element matrices reduces the computational time by $39 \%$ for the $2 \mathrm{D}$ problems and $78 \%$ for the 3D problems compared to numerical integration. The linear flux formulation also yields slightly higher accurate results compared to the conventional finite element formulation.

Four thermal and structural problems were analyzed, and the results compare favorably with available analytical and conventional finite element results. The examples have demonstrated the viability of the approach to improve the disciplinary analysis efficiency for practical steady-state thermal-structural problems.
Appendix

\section{Closed Form Finlte Element Matrlces}

The closed form expressions of the finite element matrices for two-dimensional quadrilateral element, such as the $\left[D_{x}\right]$ matrix, shown in Eq. (7), are

$$
\begin{aligned}
& D_{x}(1,1)=-D_{x}(3,3)=-\left(y_{4}-y_{2}\right) / 6 \\
& D_{x}(2,2)=-D_{x}(4,4)=-\left(y_{1}-y_{3}\right) / 6 \\
& D_{x}(1,3)=-D_{x}(3,1)=-\left(y_{4}-y_{2}\right) / 12 \\
& D_{x}(2,4)=-D_{x}(4,2)=-\left(y_{1}-y_{3}\right) / 12 \\
& D_{x}(1,2)=-\left(y_{4}+y_{3}-2 y_{2}\right) / 12 \\
& D_{x}(1,4)=-\left(2 y_{4}-y_{3}-y_{2}\right) / 12 \\
& D_{x}(2,1)=\left(y_{4}+y_{3}-2 y_{1}\right) / 12 \\
& D_{x}(2,3)=-\left(y_{4}-2 y_{3}+y_{1}\right) / 12 \\
& D_{x}(3,2)=\left(y_{4}-2 y_{2}-y_{1}\right) / 12 \\
& D_{x}(3,4)=\left(2 y_{4}-y_{2}-y_{1}\right) / 12 \\
& D_{x}(4,1)=-\left(y_{3}+y_{2}-2 y_{1}\right) / 12 \\
& D_{x}(4,3)=-\left(2 y_{3}-y_{2}-y_{1}\right) / 12
\end{aligned}
$$

where $x i$ and $y i, l=1, \ldots, 4$ are nodal coordinates of the element based on the element node numbering scheme shown in Fig. 1.

The element flux variation and the element nodal variables are related through the matrices $\left[P_{x}\right]$ and $\left[P_{y}\right]$ given in Eq. (11). The matrix, $\left[P_{X}\right]$, as an example, is given by

$$
\begin{aligned}
P_{x}(1,1)=\left(y_{2}-y_{4}\right) / 4 V_{1} \\
P_{x}(1,2)=\left(y_{4}-y_{1}\right) / 4 V_{1} \\
P_{x}(1,4)=\left(y_{1}-y_{2}\right) / 4 V_{1} \\
P_{x}(2,1)=\left(y_{2}-y_{3}\right) / 4 V_{2} \\
P_{x}(2,2)=\left(y_{3}-y_{1}\right) / 4 V_{2} \\
P_{x}(2,3)=\left(y_{1}-y_{2}\right) / 4 V_{2} \\
P_{x}(3,2)=\left(y_{3}-y_{4}\right) / 4 V_{3} \\
P_{x}(3,3)=\left(y_{4}-y_{2}\right) / 4 V_{3} \\
P_{x}(3,4)=\left(y_{2}-y_{3}\right) / 4 V_{3} \\
P_{x}(4,1)=\left(y_{3}-y_{4}\right) / 4 V_{4} \\
P_{x}(4,3)=\left(y_{4}-y_{1}\right) / 4 V_{4} \\
P_{x}(4,4)=\left(y_{1}-y_{3}\right) / 4 V_{4} \\
P_{x}(1,3)=P_{x}(2,4)=P_{x}(3,1)=P_{x}(4,2)=0
\end{aligned}
$$

where $V_{l} \mid=1, \ldots, 4$ are the constants shown in Eq. (22), and are given by,

$$
\begin{aligned}
& V_{1}=\left[\left(x_{2}-x_{1}\right) y_{4}+\left(x_{1}-x_{4}\right) y_{2}+\left(x_{4}-x_{2}\right) y_{1}\right] / 4 \\
& V_{2}=\left[\left(x_{2}-x_{1}\right) y_{3}+\left(x_{1}-x_{3}\right) y_{2}+\left(x_{3}-x_{2}\right) y_{1}\right] / 4 \\
& V_{3}=\left[\left(x_{3}-x_{2}\right) y_{4}+\left(x_{2}-x_{4}\right) y_{3}+\left(x_{4}-x_{3}\right) y_{2}\right] / 4 \\
& V_{4}=\left[\left(x_{3}-x_{1}\right) y_{4}+\left(x_{1}-x_{4}\right) y_{3}+\left(x_{4}-x_{3}\right) y_{1}\right] / 4
\end{aligned}
$$




\section{References}

1. Wieting, A. R. , Dechaumphai, P., Bey, K. S., Thornton, E. A. and Morgan, K., "Application of Integrated Fluid-Thermal-Structural Analysis Method," Presented at the 16th Congress of the International Council of the Aeronautical Sciences, Jerusalem, Israel, August 28- September 2, 1988.

2. Dechaumphai, P., Thornton, E. A. and Wieting, A. R., "Flow-Thermal-Structural Study of Aerodynamically Heated Leading Edges," AIAA Paper No. 88-2245-CP, April 1988.

3. Huebner, K. H. and Thornton, E. A., The Finite Element Method for Engineers, Wiley, Now York. 1982.

4. Reddy, J. N., An Introduction to the Finite Element Method, McGraw-Hill, New York, 1984.

5. The Mathlab Group, MACSYMA Reference Manual, The Mathlab Group Laboratory for Computer Science, MIT, Version 9, Second Printing, December 1977.

6. Boley, B. A. and Weiner, J. H., Theory of Thermal Stresses, Wiley, Now York, 1960.

7. Thornton, E. A. and Dechaumphai, P., "Coupled Flow, Thermal, and Structural Analysis of Aerodynamically Heated Panels," Journal of Aircraft, Vol. 25, No. 11, November 1988, pp. $1052-$ 1059.

8. Dechaumphai, P., Wieting, A. R. and Pandey, A. K., "Flow-Thermal-Structural Interaction of Aerodynamically Heated Leading Edges," AIAA Paper No. 89-1227-CP, April 1989.

9. Metals Handbook Committee, Metals Handbook, Eighth Edition, American Society for Metals, Ohio, 1975.

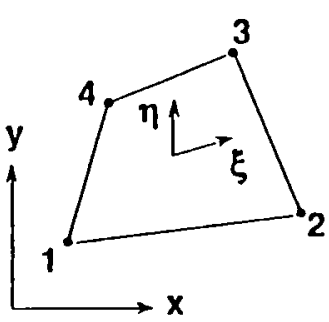

(a) Global coordinates

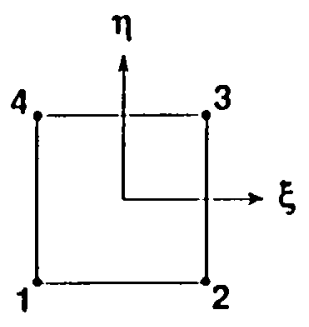

(b) Natural coordinates

Flg. 1 Quadrilateral finite element in Iwo-dimensional global and natural coordinales.

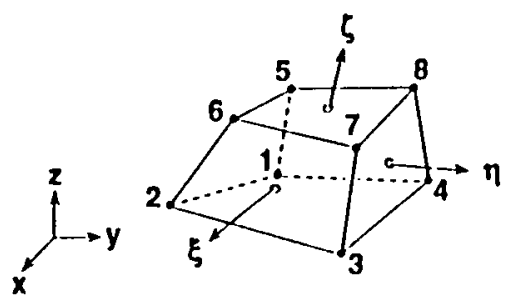

(a) Global coordlnates

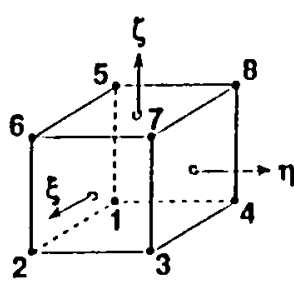

(b) Natural coordinates

Fig. 2 Hexahedral finite element in three-dimensional global and nalural coordinates.

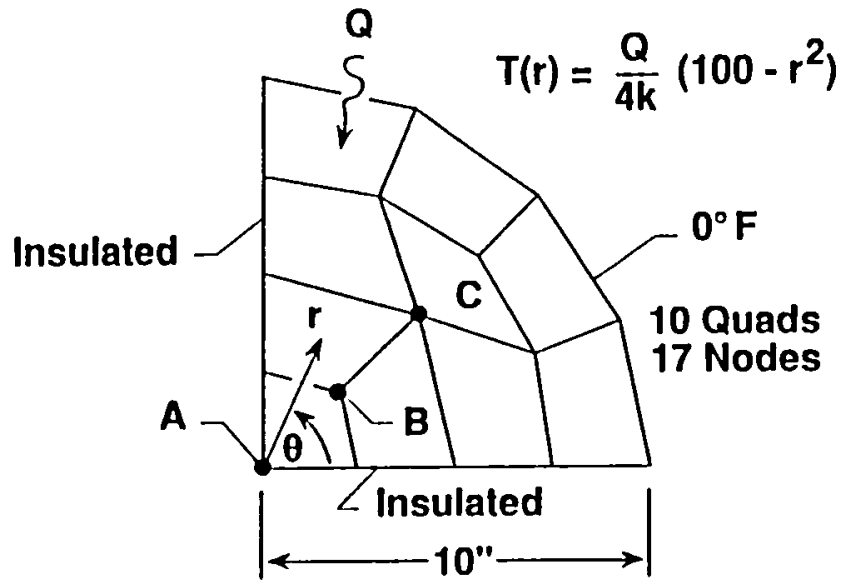

Fig. 3 Finite element model tor a 10-inch radius circular plate subjected to internal heat generalion.

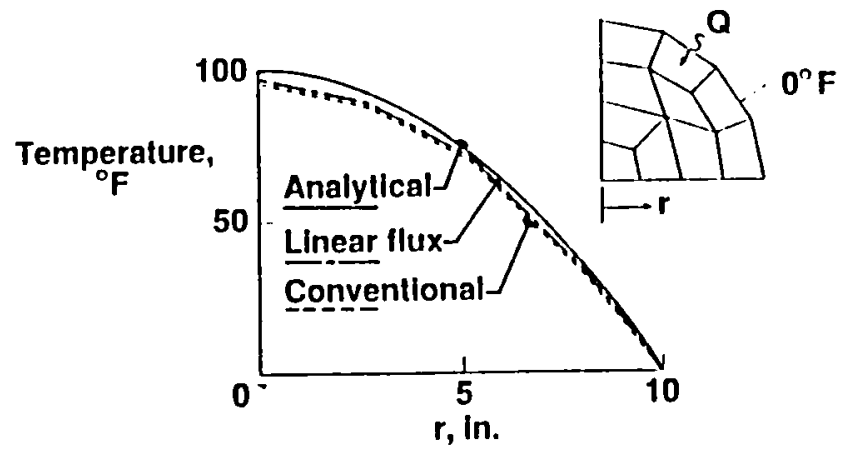

Flg. 4 Comparallve temperalure distribution for 10Inch radlus clrcular plate subjected to Internal heal generation. 


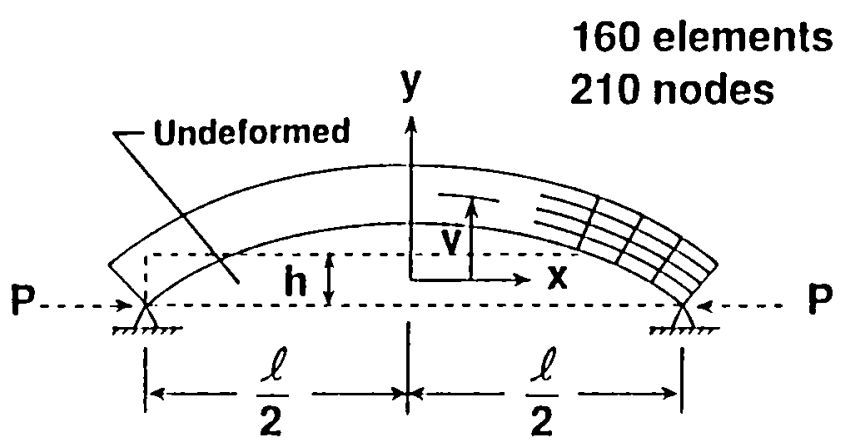

Fig. $5 \wedge$ schematic finile element model of heated beam with boundary conditions.

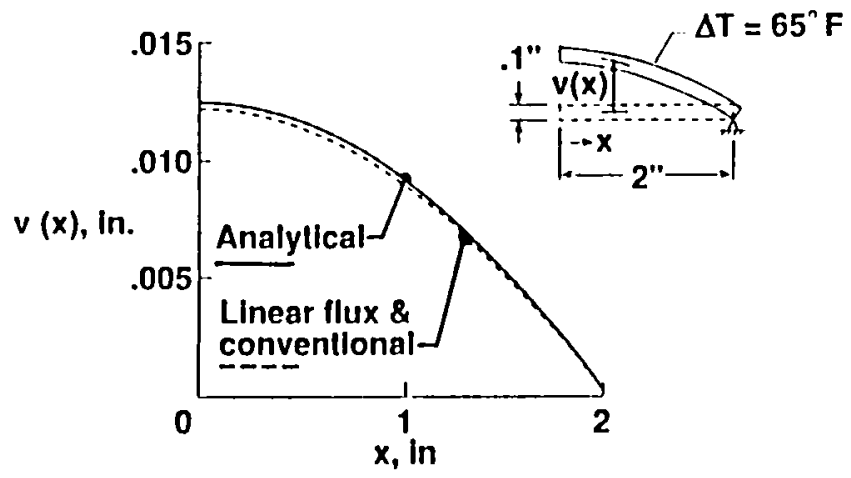

Fig. 6 Comparative defleclions for a 4 in. long beam subjected to uniform temperature increment of $65^{\circ} \mathrm{F}$.

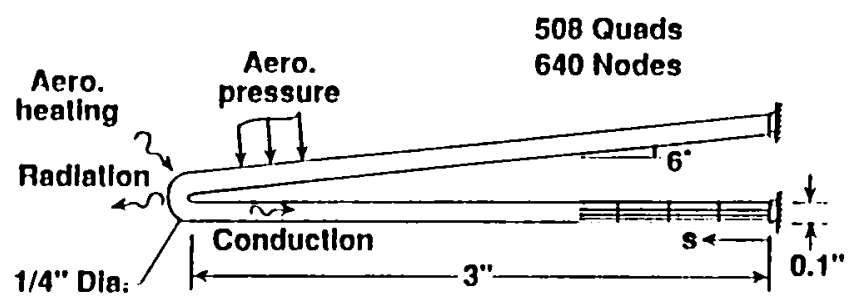

Fig. 7 A schematic thermal-structural finite element model of 0.25 -Inch diameler, 3-Inch long leading odge with boundary conditions.

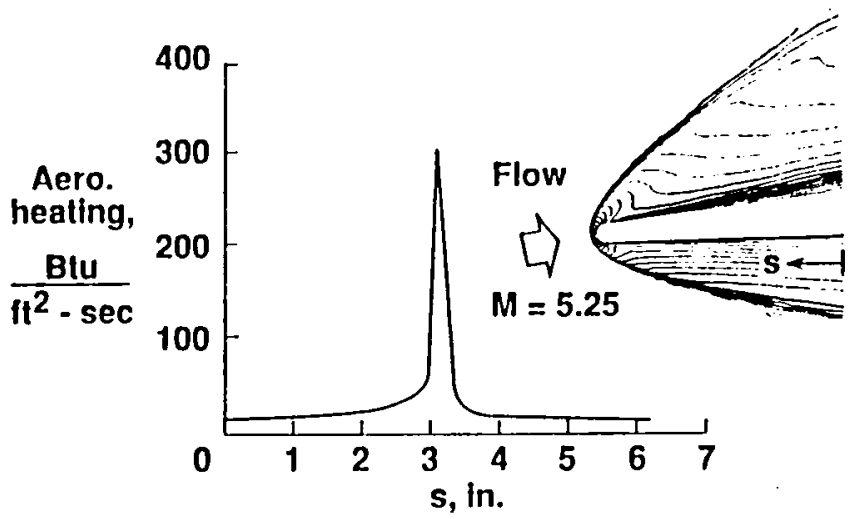

Fig. 8 Surlace heating rate distribulion and How Mach number contours for an undisturbed Mach $\mathbf{5 . 2 5}$ flow over leading edge.

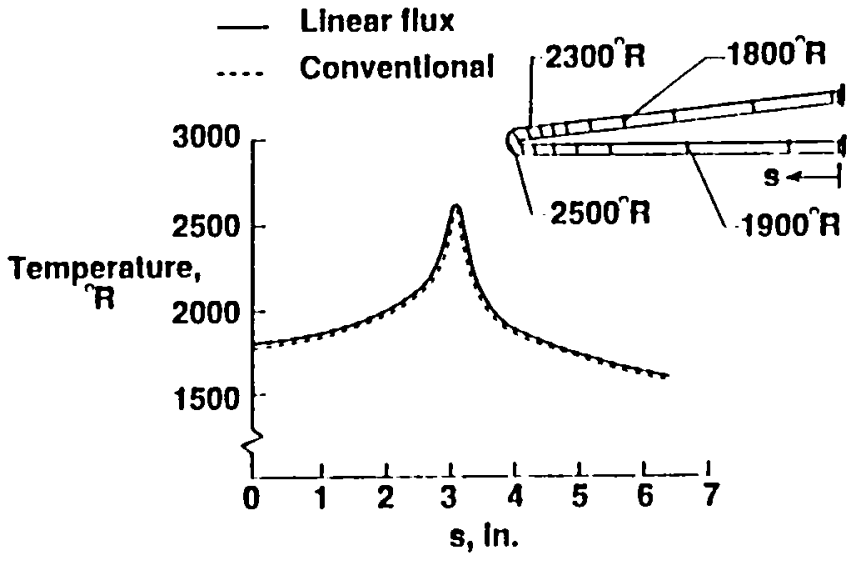

Fig. 9 Sleady-slate surlace temperalure distribulions and leading edge temperature contours.

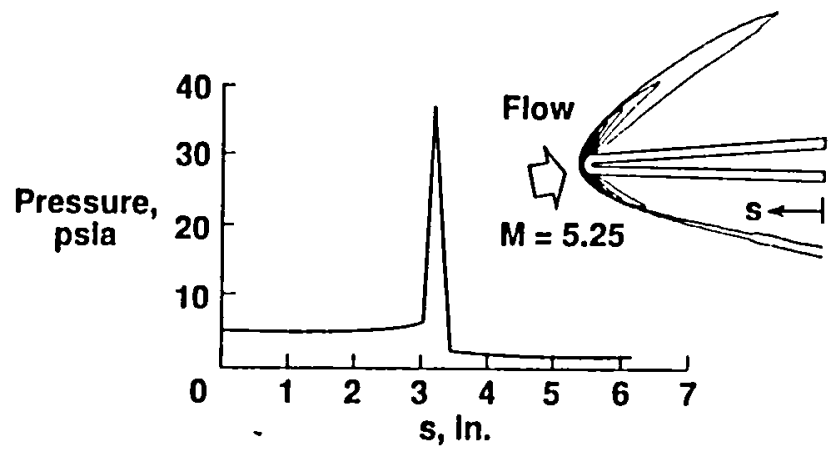

Fig. 10 Surface pressure distribullon and lilow pressure contours for an undisfurbed Mach 5.25 flow over leading edge. 


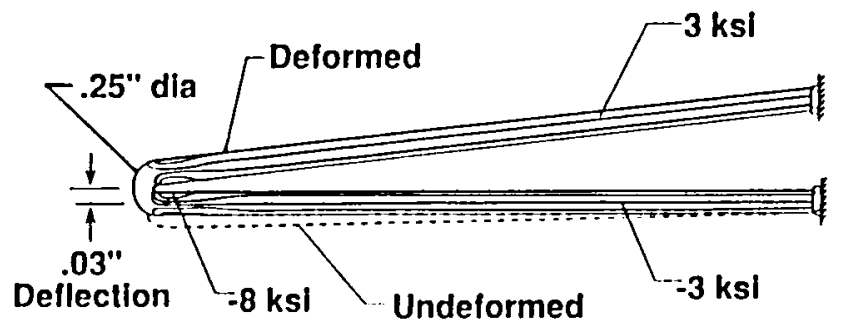

Fig. 11 Tangentlal stress contours on delormed leading edge.

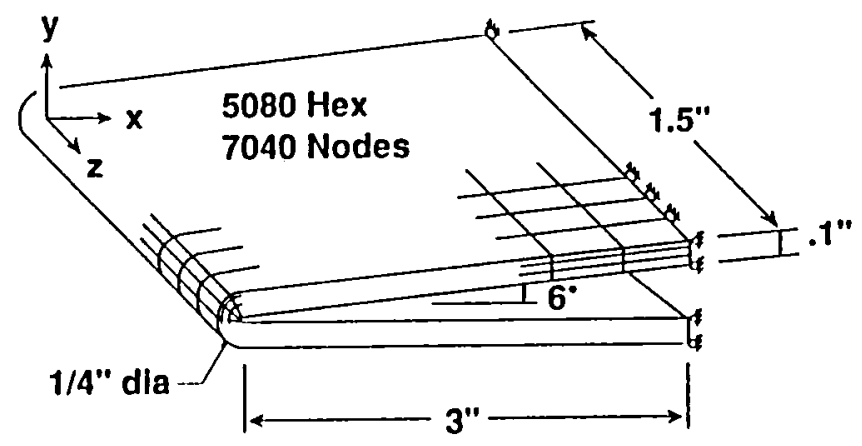

Fig. 12 A schematic three-dimensional finite element model of 0.25 -inch diameter, 3-inch long, 1.5-inch wide leading edge with boundary conditions.

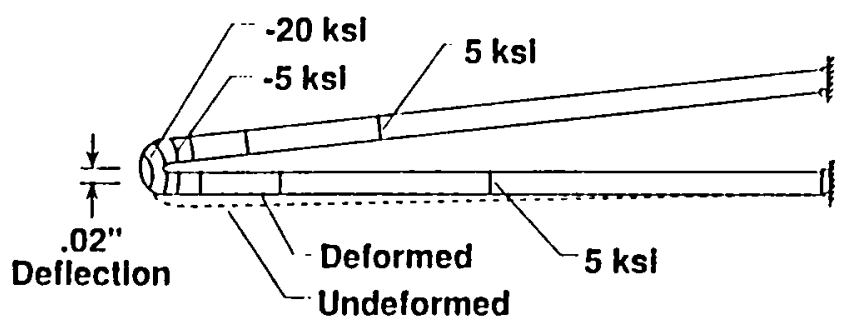

Fig. 13 Axlal stress contours on delormed leading edge. 
. 


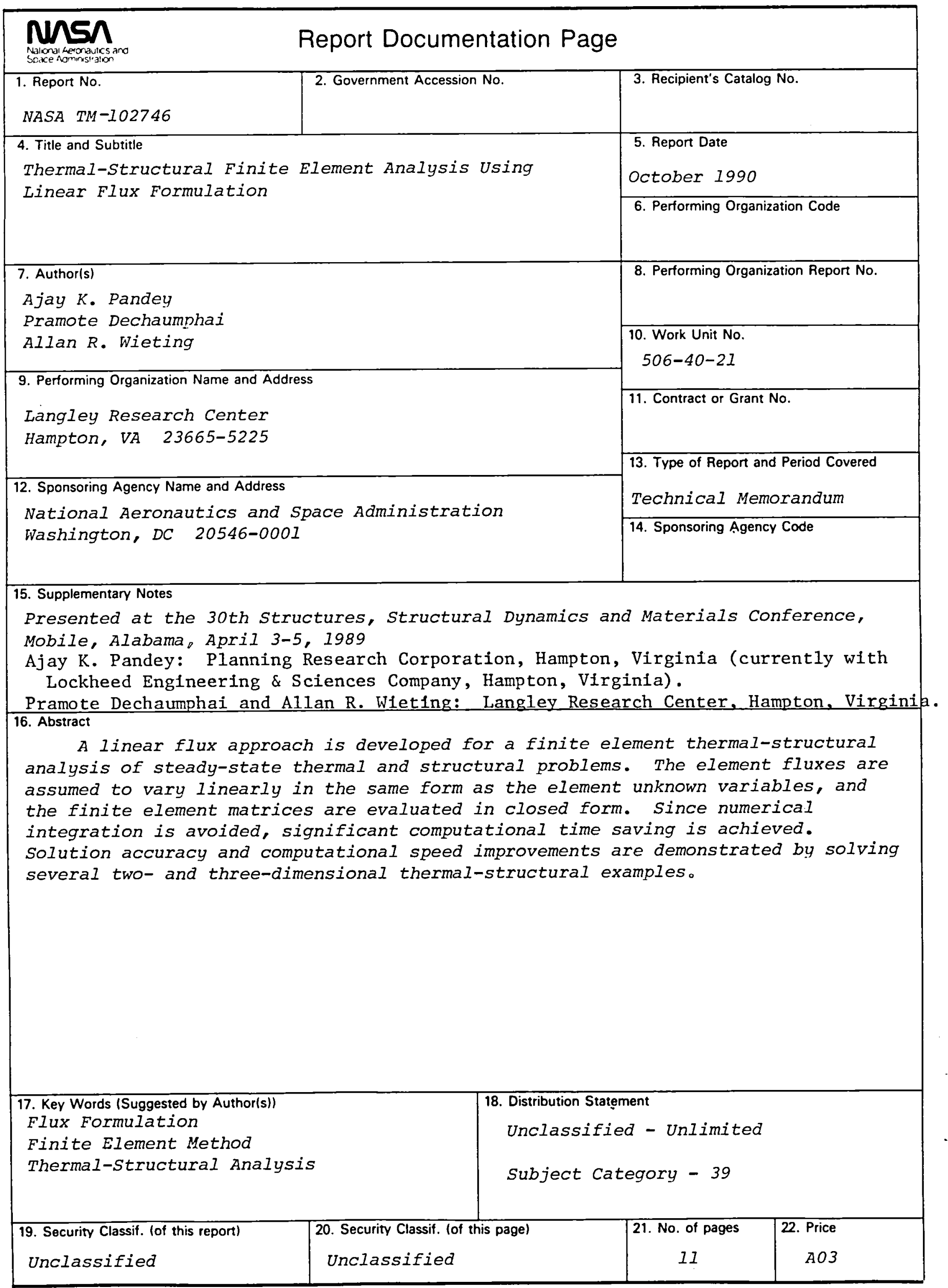




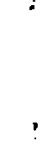


